\section{Biomarkers of dementia: from bench to clinical side}

\author{
Patrizia Mecocci, Lucia Paolacci, \\ Virginia Boccardi
}

Institute of Gerontology and Geriatrics, Department of Medicine, University of Perugia, Italy

\begin{abstract}
To date Alzheimer's dementia (AD) is defined biologically, by neuropathologic change, and clinically treating cognitive impairment as a symptom of the disease rather than the definition of the disease. This approach underlines the complexity of such a disease and should enhance efforts to identify a sensitive but easy to get biomarker that will play a key role when innovative and efficacious treatment for $\mathrm{AD}$ will be found because, then it will be possible to treat this disease before the onset of clinical symptoms. Several biomarkers have been studied in cerebrospinal fluid: amyloid beta 1-42 (A $\beta 1-42)$, total tau (t-tau), phospho-tau (ptau), $A \beta 1-42 / \mathrm{t}$ - tau ratio and $A \beta 1-42 / \mathrm{p}$-tau ratio are currently revealed in clinical practice. In the next future, it would be useful to dose biomarkers in less invasive samples (such as blood or urine) as like as to use OMICs technologies, including proteomics and metabolomics, to find more predictive and diagnostic biomarkers for $\mathrm{AD}$.
\end{abstract}

\section{Introduction}

Diagnosis of dementia should be set as soon as possible to allow the most appropriate treatment and the use of sensitive biomarkers - defined as indicators of normal biological processes, pathogenic processes, or pharmacologic responses to a therapeutic intervention that is objectively measured to support the diagnosis. The ideal biomarker should be: i) sensitive and specific of almost $80 \%$; ii) have a positive predictive value (PPV or the probability that subjects with positive screening test truly have disease-) of almost 90\%; iii) reliable, reproducible and repeatable; iv) strictly related to the pathophysiological process; v) able to set an early and differential diagnosis; vi) cheap and slightly invasive. The last published Alzheimer's dementia (AD) criteria included cerebrospinal fluid (CSF) and neuroimaging markers to improve the diagnostic accuracy, early and differential diagnosis between several dementia types and to predict the conversion from the prodromal stage to full-blown dementia. CSF biomarkers are represented by amyloid beta 1-42 (A $\beta 1-42)$, total tau (t-tau), phospho-tau (ptau), $A \beta 1-42 / \mathrm{t}-$ tau ratio and $A \beta 1-42 / \mathrm{p}$-tau ratio. Their diagnostic accuracy is shown in Table 1. To date, the use of biomarkers is based more on practical considerations that reflect resources and experience, rather than on clinical and evidence-based considerations. European guidelines state that they are rated as class II and class III of evidence - i.e., slightly supportive - respectively for positive and differential diagnosis of ADwith some difficulties related to a different reimbursement through different countries.

\section{Biomarkers in clinical practice from healthy subjects through mild cognitive impairment}

To detect cognitive impairment in prodromal or early stages, the use of biomarkers as a screening tool for apparently healthy individuals is still under debate. Firstly because it is not feasible to accurately identify all individuals with prodromal dementia with the sole recruitment of general practice physicians, as demonstrated in the UK, where such an attempt was phased out in two years. ${ }^{1}$ Secondly, because the clinical course of dementia is not yet amenable to intervention since currently there is no curative drug for such a disease. Therefore, the use of diagnostic biomarkers in the absence of efficacious treatments able to cure or to delay disease progression, do not make available a population screening.

Another problematic issue is the usefulness of CSF biomarkers in mild cognitive impairment (MCI), a condition of cognitive decline without interference with activities of daily life, with a wide range of prevalence $(5-37 \%)$, due to changes in criteria and differences in populations studied and methodology. ${ }^{2}$ Published studies show accuracy for A $\beta 1-42$, t-tau, and p-tau in detecting prodromal AD subjects up to $90 \%$ $(93.5 \%)$ of sensibility and $80 \%(82.7 \%)$ of specificity, but data are widely variable. ${ }^{3}$ To have an appropriate evaluation it might be essential to know the predictive value (PV) that depends also from prevalence of disease: if the prevalence is low in general population, predictive value will be lower than specificity and sensibility values, hence if prevalence is 5\% PV will be lower than in case of $37 \%$ of prevalence of disease. However, the CSF mentioned above biomarkers is likely to predict the clinical
Correspondence: Patrizia Mecocci, Institute of Gerontology and Geriatrics, Department of Medicine, University of Perugia, Piazzale

Gambuli 1, 06132 Perugia, Italy.

Tel.: +39.0755.783270.

E-mail: patrizia.mecocci@unipg.it

Key words: Alzheimer's dementia; cerebrospinal fluid biomarkers; plasma biomarkers; metabolomics.

Conflict of interest: the authors declare no potential conflict of interest.

Received for publication: 25 July 2018.

Accepted for publication: 20 August 2018.

This work is licensed under a Creative Commons Attribution-NonCommercial 4.0 International License (CC BY-NC 4.0).

CCopyright P. Mecocci et al., 2018

Licensee PAGEPress, Italy

Geriatric Care 2018; 4:7718

doi:10.4081/gc.2018.7718

progression of AD. Sierra-Rio and coll. ${ }^{4}$ found that MCI and subjective cognitive decline (SCD) individuals with abnormal $\mathrm{A} \beta 42 /$ phosphorylated tau ratio had a higher proportion of conversion to dementia during 5-year follow-up, supporting the utility of AD CSF biomarkers to predict a clinical decline in subjects with SCD or MCI in the medium term. On the other hand, the normality of AD CSF biomarkers could exclude progression to $\mathrm{AD}$ dementia. Although we do not have therapeutic tools for the disease, this prognostic information might have clinical relevance in subjects seeking answers when attending a specialist setting. ${ }^{4}$

Interlaboratory and interlaboratory variability in dosing CSF biomarkers represent a critical problematic issue to define their accuracy. Therefore different efforts on biomarkers harmonization studies have been made with the introduction of novel assays to provide a minimal lot-to-lot variation and thus leading to a higher agreement between different centers and measurements.

Finally, another limitation is represented by CSF biomarkers usefulness in the oldest olds, the part of population aged 85 years or more, who has been growing very fast in last decades reaching more than $1 \%$ of the Italian population. They are an extremely heterogeneous group, also according to the clinical and neuropathological presentation of dementia. In fact, oldest olds can be classified as: i) escapers (who reach 100 years and more without diseases); ii) delayers (who start to be affected by chronic diseases after 85 years old); iii) and 
survivors (who survive together with their chronic diseases after 85 years old). ${ }^{5}$ Despite the presence of classical neuropathological hallmarks, oldest old subjects often preserve their cognitive performances. It is unclear if they had better tolerate the adverse effects of neuropathological alterations or if they do not live long enough to express their clinically visible effects. Mattson and coll. ${ }^{6}$ showed that the diagnostic accuracy of CSF biomarkers for AD decreases with age; nevertheless the negative (NPV) predictive values remains persistently high also in oldest old, allowing to rule out $\mathrm{AD}$ even in this class of age.

However, biomarkers have beneficial and potential use in the oldest old population, mainly with the evaluation of the progression from MCI to dementia. Adding CSF biomarkers to the usual care diagnostic workup can improve the ability to differentiate between subjects with or without progression to dementia, ${ }^{7}$ especially for escapers, who might also benefit of their strong negative predictive value. In fact, the exclusion of $\mathrm{AD}$ pathology in a well-fit subject older than 85 years means that he could spend the rest of life without the fear of dementia, improving quality of life. Conversely, fit oldest old subjects with the mild cognitive decline with positive biomarkers could be included in clinical trials that currently exclude the oldest olds. In survivors and delayers, the medical practice should be performed according to ethical principles of beneficence, autonomy, justice, integrity, dignity, and vulnerability, so that it is often dispensable to make an accu- rate diagnosis or to predict MCI conversion in these classes.

\section{Pathogenesis of dementia: toward new markers}

CSF biomarkers limitations are probably due to an erroneous rationale behind dementia pathogenesis: amyloid and tau hypothesis have been widely studied in recent years, but according to several studies, they should represent a final stage of neuronal damage rather than the primary and only cause of neurodegeneration. Furthermore, Giuffrida and coll. ${ }^{8}$ even showed that $A \beta 1-42$ monomers have a broad neuroprotective activity related to insulin/IGF-1 signaling. Many other hypotheses have been proposed, and they could contribute all together to dementia pathogenesis (Table 2). Indeed, lumbar puncture is a safe procedure but it is quite invasive and expensive, so it would be more comfortable and cheaper to obtain diagnostic biomarkers from blood taking advantage of new methodologies such as proteomic, lipidomic, and genomic profiling.

\section{New potential cerebrospinal fluid biomarkers \\ Cerebrospinal fluid visinin-like-protein-1}

Visinin-like-protein-1 (VILIP-1) belongs to the family of visinin-like proteins, which are neuronal calcium sensor proteins, and it is implicated in both neuroprotective and neurotoxic functions. In par- ticular, VILIP-1 is released into the CSF from injured neurons and in neurodegenerative diseases. Tarawneh and coll. ${ }^{9}$ showed a role of this protein as a diagnostic and prognostic marker of $\mathrm{AD}$ in subject aged 38-93 years old.

\section{Neurogranin and $Y K L-40$}

Neurogranin is a marker of synaptic dysfunction that may be an early pathologic process in age-related neurodegeneration, and a sensitive marker of age-related cognitive abilities, potentially preceding or even acting independently from AD pathogenesis. YKL-40 (chitinase-3 like-1) is a marker of neuroinflammation, and it is up-regulated in various inflammatory conditions and expressed by neutrophils, macrophages, chondrocytes, and vascular smooth muscle cells and astrocytes. Their CSF levels are increased in $\mathrm{AD}$, and decreased in Parkinson dementia or dementia with Lewy Body; moreover, the first one seems to be reduced in vascular dementia and Frontotemporal dementia, and the latter appears to be increased in CSF of people with frontotemporal dementia. ${ }^{10,11}$ Neurogranin detection along with the $\beta$-site amyloid precursor protein cleaving enzyme 1 (BACE1) (i.e., the enzyme that catalyzes the first step in the formation of an amyloid beta peptide from amyloid precursor protein in AD brain) improves diagnostic performance rather than neurogranin alone. De Vos and coll. ${ }^{11}$ showed that NGRN/BACE1 ratio was well related with the yearly decline in mini-mental state examination (MMSE) scores in patients with MCI and AD.

Table 1. The accuracy of available cerebrospinal fluid biomarkers..$^{22,23}$

\begin{tabular}{|c|c|c|c|}
\hline Biomarker & Specificity (95\% CI) & Sensitivity (95\% CI) & PPV \\
\hline $\mathrm{A} \beta 1-42$ & $0.58-0.80$ & $0.55-0.82$ & $0.48-0.92$ \\
\hline t-tau & $0.61-0.96$ & $0.60-0.98$ & $0.57-0.98$ \\
\hline p-tau & $0.76-0.86$ & $0.82-0.92$ & $0.86-0.94$ \\
\hline A 1-42/t- tau ratio & $0.38-0.86$ & $0.71-0.98$ & $0.57-1.00$ \\
\hline A 1-42/p-tau ratio & $0.89-0.96$ & $0.74-0.86$ & $0.83-0.91$ \\
\hline
\end{tabular}

$\mathrm{CI}$, confidence interval; PPV, positive predictive value.

Table 2. The pathogenic hypothesis of Alzheimer's dementia. ${ }^{24}$

\begin{tabular}{|c|c|}
\hline Hypothesis & Mechanism \\
\hline Inflammatory hypothesis & Microglial activation, pro-inflammatory cytokines release, insulin/IGF-1 resistance \\
\hline Oxidative hypothesis & Radical oxidative species exposure, cellular oxidative stress, protein oxidation, protein nitration, glycoxidation and lipid peroxidation. \\
\hline Cholinergic hypothesis & Degeneration of cholinergic neurons, reduction of cholinergic tone in the cortex and hippocampus, cognitive dysfunction \\
\hline Insulin resistance & Impairments of insulin/IGF-1 signaling and glucose metabolism, impaired synaptic plasticity, synaptic degeneration, cell death \\
\hline Gut microbiota activity & $\begin{array}{l}\text { Interactions with the central nervous system through direct and indirect pathways involving vagal nerve activation, cytokine } \\
\text { production, and release of neuropeptide/neurotransmitters and short-chain fatty acids }\end{array}$ \\
\hline
\end{tabular}




\section{AD7c-NTP}

NTP (neuronal thread proteins) are a family of proteins expressed in brain and some neuroectodermal tumor cell lines; ADassociated NTP (AD7c-NTP) seems to be elevated in brain tissue, cerebrospinal fluid, and even in urine of patients with Alzheimer's disease reflecting the severity and progression of dementia as demonstrated in several recent studies. ${ }^{2,12}$ The high accuracy of urine measurement (sensibility of $89 \%$ and specificity of 85\%) makes of AD7c-NTP a promising biomarker of dementia.

\section{Microbiota biomarkers}

Gut microbes can produce secretory products as amyloids, lipopolysaccharides, virulence factors rhamnolipids (RLs), toxins, and other neuroactive compounds; in particular elevated RLs, levels have been found in cerebrospinal fluid of both $\mathrm{AD}$ and MCI patients compared to healthy. Moreover, they seem to be related to the AD stages clinical severity. ${ }^{13}$

\section{Blood biomarkers AB1-42/AB1-40 ratio}

Findings on the relationship between $\mathrm{AD}$ pathogenesis and plasmatic $\mathrm{A} \beta$ levels are contradictory, but new elaborate techniques revealed a correlation between an increase in the plasma $A \beta 42 / A \beta 40$ ratio and risk of developing AD. ${ }^{14}$ Fei and coll. ${ }^{15}$ also found a link between this ratio and the risk of progression from $\mathrm{MCI}$ to $\mathrm{AD}$ with a specificity of $70 \%$ and sensibility of $85 \%$.

\section{Biomarkers of neocortical amyloid burden (NAB): the fibrinogen gamma chain}

It represents the gamma component of fibrinogen, produced by FGG, a human gene found on chromosome 4. It can predict high NAB when combined with age, yielding a sensitivity of $59 \%$ and specificity of $78 \%{ }^{16}$ that increase respectively to $71 \%$ and $84 \%$ if combined with a 4-plex metabolic panel (phosphatidylcholine, PE 39:7, anandamide, and anandamide isotope). ${ }^{17}$

\section{Clusterin}

Clusterin is a protein overexpressed in the brain of $\mathrm{AD}$ patients associated with the clearance of cellular debris and apoptosis. It has been demonstrated that MCI patients have higher plasmatic clusterin levels compared to healthy controls; moreover higher clusterin levels were associated with significantly lower MMSE scores at baseline and with the longitudinally structural atrophy for patients with MCI. ${ }^{18}$

\section{Neurofilament}

It is a protein of neuronal cytoskeleton where it provides mechanical strength and regulates axonal diameter; its levels are higher in AD, FTD and Parkinsonism compared to healthy control, also in oldest olds, and it is related to brain atrophy. ${ }^{19}$

\section{Metabolic biomarkers}

Lipidomics research involves the identification and quantification of cellular lipid molecular species and their interactions with other lipids, proteins, and different metabolites. Extensively studied lipidomic biomarkers of AD include abnormal glycerophospholipids (due to an abnormality in the integrity of cell membranes). Notably, Mapston and coll. ${ }^{20}$ reported a set of 10 phospholipids from peripheral blood that predicted phenoconversion to either aMCI or AD within 2-3 years, with over $90 \%$ accuracy.

\section{Micro-RNA}

mi-RNA is endogenous $\sim 23$-nucleotide non-coding RNA molecules highly conserved in eukaryotes that regulate gene expression through post-transcriptional repression. Deregulation in their expression modulate some AD-related genes (such as $\mathrm{A} \beta, \mathrm{BACE} 1$, tau, $\alpha$ and $\gamma$ secretase genes) and promotes disease progression affecting levels of $\mathrm{A} \beta$, p-tau and synaptic damage. Recently Reddy and coll. ${ }^{21}$ reviewed the role as potential biomarkers of miRNAs in blood and CSF from patients with $\mathrm{AD}$ showing neuroprotective forms (e.g., miRNAs 101, 124, 219, 16) and neurodegenerative forms (e.g., RNAs-26b, 206, $125,33)$ in the brain and hippocampus..$^{22-24}$

\section{Conclusions}

So far it is corroborated the measurement of CSF classical biomarkers (CSF $\mathrm{A} \beta 42$, t-tau, and p-tau) in clinical practice, but they are not entirely suitable for AD diagnosis showing several limitations, such as variability inter and intra-laboratory, lack of universal cut-off, partial usefulness in MCI and oldest olds, absence of indication in healthy subjects screening. Hence, efforts are needed to find novel candidates in CSF, or in more suitable and easy to get samples including blood or urine. In the next future, it is hopeful that research will find easily measurable biomarkers that can predict cognitive decline in subjects who have a preclinical, prodromal, or clinical AD.

\section{References}

1. Chambers LW, Sivananthan S, Brayne C. Is dementia screening of apparently healthy individuals justified? Adv Prev Med 2017;2017:9708413.

2. Sachdev PS, Lipnicki DM, Kochan NA, et al. The prevalence of mild cognitive impairment in diverse geographical and ethnocultural regions: The COSMIC Collaboration. PLoS ONE 2015;10: e0142388.

3. Sheng C, Huang Y, Han Y. Dissection of prodromal Alzheimer's disease. Front Biosci (Landmark Ed) 2018;23:1272-91.

4. Sierra-Rio A, Balasa M, Olives J, et al. Cerebrospinal fluid biomarkers predict clinical evolution in patients with subjective cognitive decline and mild cognitive impairment. Neurodegener Dis 2016;16:69-76.

5. Evert J, Lawler E, Bogan H, Perls T. Morbidity profiles in centenarians: survivors, delayers, and escapers. J Gerontol A Med Sci 2003;58:232-7.

6. Mattsson N, Rosen E, Hansson O, et al. Age and diagnostic performance of Alzheimer disease CSF. Neurology 2012;78:468-76.

7. Handels RLH, Vos SJB, Kramberger $\mathrm{MG}$, et al. Predicting progression to dementia in persons with mild cognitive impairment using cerebrospinal fluid markers. Alzheimers Dement 2017;13: 903-12.

8. Giuffrida ML, Tomasello MF, Pandini G, et al. Monomeric $\beta$-amyloid interacts with type-1 insulin-like growth factor receptors to provide energy supply to neurons. Front Cell Neurosci 2015;9: 297.

9. Tarawneh R, D’Angelo G, Macy E, et al. Visinin-like protein-1: diagnostic and prognostic biomarker in Alzheimer disease. Ann Neurol 2011;70:274-85.

10. Janelidze S, Hertze J, Zetterberg H et al. Cerebrospinal fluid neurogranin and YKL-40 as biomarkers of Alzheimer's disease. Ann Clin Transl Neurol 2015;3:12-20.

11. De Vos A, Struyfs H, Jacobs D, et al. The cerebrospinal fluid neurogranin/BACE1 ratio is a potential correlate of cognitive decline in Alzheimer's disease. J Alzheimers Dis 2016;53:1523-38.

12. Ma L, Chen J, Wang R, et al. The level of Alzheimer-associated neuronal thread protein in urine may be an important biomarker of mild cognitive impairment. J Clin Neurosci 2015;22:649-52.

13. Andreadou E, Pantazaki AA, Daniilidou M, Tsolaki M. Rhamnolipids, microbial virulence factors, in Alzheimer's disease. J Alzheimers Dis 2017;59:209-22.

14. Nakamura A, Kaneko N, Villemagne VL, et al. High-performance plasma amyloid- $\beta$ biomarkers for Alzheimer's disease. Nature 2018;55:249-54. 
15. Fei M, Jianghua W, Rujuan M, et al. The relationship of plasma $A \beta$ levels to dementia in aging individuals with mild cognitive impairment. J Neurol Sci 2011;305:92-6.

16. Ashton NJ, Kiddle SJ, Graf J, et al. Blood protein predictors of brain amyloid for enrichment in clinical trials? Alzheimer's Dement 2015;1:48-60.

17. Voyle N, Kim M, Proitsi P, et al. Blood metabolite markers of neocortical amyloid- $\beta$ burden: discovery and enrichment using candidate proteins. Transl Psychiatry 2016;6:e719.

18. Hsu JL, Lee WJ, Liao YC, et al. The clinical significance of plasma clusterin and $A \beta$ in the longitudinal follow-up of patients with Alzheimer's disease. Alzheimers Res Ther 2017;9:91.

19. Pereira JB, Westman E, Hansson O. Association between cerebrospinal fluid and plasma neurodegeneration biomarkers with brain atrophy in Alzheimer's disease. Neurobiol Aging 2017;58:14-29.

20. Mapstone M, Cheema AK, Fiandaca MS, et al. Plasma phospholipids identify antecedent memory impairment in older adults. Nat Med 2014;20:415-8.

21. Reddy PH, Tonk S, Kumar S, et al. A critical evaluation of neuroprotective and neurodegenerative MicroRNAs in
Alzheimer's disease. Biochem Biophys Res Commun 2017;483:1156-65.

22. Huynh RA, Mohan C. Alzheimer's Disease: biomarkers in the genome, blood, and cerebrospinal fluid. Front Neurol 2017;8:102.

23. Lehmann S, Gabelle A, Paquet C. Can we rely only on ratios of cerebrospinal fluid biomarkers for AD biological diagnosis? Alzheimers Dement 2015;11: 1125-26.

24. Du X, Wang X, Geng M. Alzheimer's disease hypothesis and related therapies. Transl Neurodegener 2018; 7:2. 